\title{
Prefrontocortical Dopamine Loss in Rats Delays Long-Term Extinction of Contextual Conditioned Fear, and Reduces Social Interaction Without Affecting Short-Term Social Interaction Memory
}

\author{
Emilio Fernandez Espejo*,1 \\ 'Departamento de Fisiologia Medica, Facultad de Medicina, Universidad de Sevilla, Sevilla, Spain
}

\begin{abstract}
Prefrontal dopamine loss delays extinction of cued fear conditioning responses, but its role in contextual fear conditioning has not been explored. Medial prefrontal lesions also enhance social interaction in rats, but the role of prefrontal dopamine loss on social interaction memory is not known. Besides, a role for subcortical accumbal dopamine on mnesic changes after prefrontal dopamine manipulation has been proposed but not explored. The objective was to study the involvement of dopaminergic neurotransmission in the medial prefrontal cortex (mPFC) and nucleus accumbens in two mnesic tasks: contextual fear conditioning and social interaction memory. For contextual fear conditioning, short- and long-term freezing responses after an electric shock were studied, as well as extinction retention. Regarding social interaction memory, the recognition of a juvenile, a very sensitive short-term memory test, was used. Dopamine loss was carried out by injection of 6-hydroxydopamine, and postmortem catecholamine levels were analyzed by high-performance liquid chromatography. Prefrontocortical dopamine loss ( $>76 \%)$ led to a reactive enhancement of accumbal dopamine content $(p<0.0 \mathrm{I})$, supporting the hypothesis that a hyperdopaminergic tone emerges in the nucleus accumbens after prefrontocortical dopamine loss. In lesioned rats, long-term extinction of contextual fear conditioning was significantly delayed and extinction retention was impaired without changes in acquisition and short-term contextual fear conditioning and, on the other hand, acquisition and short-term social interaction memory were not affected, although time spent on social interaction was significantly reduced. Added dopamine loss in the nucleus accumbens $(>76 \%$ ) did not alter these behavioral changes. In summary, the results of the present study indicate that the dopaminergic network in the mPFC (but not in the nucleus accumbens) coordinates the normal long-term extinction of contextual fear conditioning responses without affecting their acquisition, and it is involved in time spent on social interaction, but not acquisition and short-term social interaction memory.

Neuropsychopharmacology (2003) 28, 490-498. doi:I 0.1 038/sj.npp. 1300066
\end{abstract}

Keywords: medial prefrontal cortex; nucleus accumbens; dopamine; conditioned fear; extinction; memory

\section{INTRODUCTION}

The prefrontal cortex is known to be involved in mnesic processes, although its participation is organized independently in different prefrontocortical regions. Among these regions, the medial prefrontal cortex (mPFC) has extensive bidirectional connections with the hippocampus and amygdala, which supports a role of this structure on mnesic processes (Goldman-Rakic et al, 1984; von Hoesen et al, 1972, 1975). Thus, the medial part has been proposed to participate in short-term object memory in rats and

\footnotetext{
*Correspondence: Dr EF Espejo, Departamento de Fisiologia Medica, Facultad de Medicina, Universidad de Sevilla, Av. Sanchez Pizjuan 4, E-4I009 Sevilla, Spain, Fax: 34-95455 I769, E-mail: efespejo@us.es Received 18 March 2002; revised 19 June 2002; accepted 22 August 2002

Online publication: 20 September 2002 at http://www.acnp.org/ citations/Npp092002386
}

monkeys (Kowalska et al, 1991), and the lesion of this region is also known to interfere with the extinction of cued conditioned fear responses (Morgan et al, 1993; Morgan and LeDoux, 1995; Quirk et al, 2000), although negative results have also been reported (Gewirtz et al, 1997). The role of the mPFC in contextual conditioned fear, a type of memory that is dependent on both hippocampal and amygdala function, has been little explored, and it is known that prefrontocortical lesions do not affect acquisition and extinction of contextual conditioned fear (Morgan et al, 1993). The mPFC has also been involved in social interaction, because medial prefrontal transection is known to enhance social interaction in rats (Gonzalez et al, 2000; Tucci et al, 2000), but its role on mnesic processes during social interaction is not known.

The mPFC also plays an important role in the dopaminergic system network, since it receives input from the mesocortical dopamine (DA) system, and sends efferents to 
the nucleus accumbens, dorsal striatum, and midbrain DA cell groups (Carter and Pycock, 1978, 1980; Pycock et al, 1980; Glowinski et al, 1984; Grace, 1991; Roberts et al, 1994; Kolachana et al, 1995; Kurachi et al, 1995). Dopaminergic activity in the mPFC is quite important in the neuromodulation of the 'cognitive functions' of the prefrontal cortex. However, the mesocortical dopaminergic neuromodulation is not straightforward, because the relation between prefrontal dopamine and 'cognitive performance' in memory tests depends on the nature of the task (Roberts et al, 1998). Thus, while prefrontal dopamine loss is known to impair performance on delayed alternation or delayed response in rats and monkeys (Murphy et al, 1996), the extinction of cued conditioned fear responses is delayed (Morrow et al, 1999), which indicates that the prefrontal cortex and its dopaminergic network 'organize' differently the behavioral response to varying motivational or mnesic demands (Roberts et al, 1998). In this respect, the effects of prefrontal dopamine loss in contextual fear conditioning or social interaction memory have not been explored. Furthermore, after prefrontal dopamine manipulation, a role of the subcortical mesoaccumbal DA system on fear conditioning or social interaction changes has been proposed, since prefrontal dopamine inhibits corticofugal excitatory neurons that innervate accumbal and DA midbrain cell regions, and accumbal dopamine metabolism is enhanced during fear conditioning (Morrow et al, 1996, 1999) and social interaction (Tucci et al, 2000). However, such a role of the accumbal dopaminergic system in fear conditioning tasks or social interaction memory has not been explored. The objective of this study was to study the involvement of dopaminergic neurotransmission in the $\mathrm{mPFC}$ and nucleus accumbens in contextual fear conditioning and social interaction memory tests. For contextual fear conditioning, freezing responses after an electric shock were studied. Regarding social interaction memory, the recognition of a juvenile was used (Dantzer et al, 1987). Short-term memory, as measured through this test, is disrupted after lesions of the septohippocampal pathway and is very sensitive to cholinergic drugs (Pério et al, 1989).

\section{MATERIALS AND METHODS}

\section{Animals and Ethics}

Male Wistar rats (275-325 g) from the breeding colony of the Faculty of Medicine of the University of Seville, Spain, were singly housed in the vivarium. Laboratory temperature was kept at $22 \pm 1{ }^{\circ} \mathrm{C}$, and a 12-h light-dark cycle (lights on at $08: 00 \mathrm{~h})$ was maintained throughout the experiment. Food (lab chow) and water were available ad lib. Experiments were performed in accordance with the European Communities Council Directive for the employment of laboratory animals (86/609/EEC).

\section{Experiments and Groups}

The behavioral effects and neurochemical changes in both the $\mathrm{mPFC}$ and nucleus accumbens after prefrontal dopamine loss were studied in experiment 1. For this experiment, rats subjected to contextual fear conditioning were randomly assigned to two groups: (i) sham-lesioned rats
( $n=12$ ), and (ii) 6-OHDA-induced lesioned rats in the mPFC $(n=12)$. Each group was subdivided into two subgroups (see the 'Behavioral testing' section for more details): (i) sham rats 'with electric shock' (shocked, $n=6$ ) or 'without electric shock' (nonshocked, $n=6$ ), and (ii) lesioned rats 'with electric shock' (shocked, $n=6$ ) or 'without electric shock' (nonshocked, $n=6$ ). Those rats subjected to social interaction were randomly assigned to two groups: (i) sham-lesioned rats $(n=33)$, and (ii) 6OHDA-induced lesioned rats in the $\operatorname{mPFC}(n=32)$. Each group was subdivided into two subgroups: (i) sham rats interacting with a familiar juvenile $(n=17)$ or with an unfamiliar juvenile $(n=16)$, and (ii) lesioned rats interacting with a familiar juvenile $(n=16)$ or with an unfamiliar juvenile $(n=16)$. The behavioral and neurochemical effects of dopamine loss in both the MPFC and nucleus accumbens were studied in experiment 2 . Rats were randomly assigned to two groups: (i) sham rats (fear conditioning, $n=9$; social interaction, $n=18$ ), and (ii) 6-OHDA-induced lesioned rats in both the mPFC and nucleus accumbens (fear conditioning, $n=7$; social interaction, $n=17$ ). A valid DA loss in both $\mathrm{mPFC}$ and nucleus accumbens was considered when dopamine levels were depleted beyond $75 \%$, and $\mathrm{mPFC}$ noradrenaline (NA) contents were not significantly diminished, as measured by HPLC. Only those rats that met these criteria were selected for the statistical analysis. These animals are those where dopamine levels are reliably reduced beyond the range where compensatory mechanisms are able to restore normal dopaminergic activity, if moreover noradrenergic activity is not affected in the mPFC (Venator et al, 1999). Thus, although 59 (experiment 1) and 32 rats (experiment 2) were lesioned with 6-OHDA, only 44 (experiment 1) and 24 rats (experiment 2) fulfilled these criteria.

\section{Prefrontocortical and Accumbal Lesions with 6-OHDA}

Rats were injected with desipramine ( $15 \mathrm{mg} / \mathrm{kg}$ i.p.), $30 \mathrm{~min}$ before 6-OHDA lesion in order to protect noradrenergic terminals from 6-hydroxydopamine toxicity. Then rats were anesthetized with chloral hydrate $(425 \mathrm{mg} / \mathrm{kg}$ i.p.), and placed into a David Kopf stereotaxic apparatus with the incisor bar set $3.3 \mathrm{~mm}$ below the interaural line. After scalp incision, burr holes were drilled over the injection sites and a blunted 30-gauge cannula, connected to a $10 \mu \mathrm{l}$ Hamilton syringe, was lowered to the injection site. The following coordinates were used: $\mathrm{AP}=+3.5 \mathrm{~mm}$ with respect to bregma, $\mathrm{L}= \pm 0.8, \mathrm{~V}=-3.3,-4.3$ (Paxinos and Watson, 1997). At each of the two injection sites (corresponding to the prelimbic and infralimbic cortex, respectively), $1 \mu \mathrm{l}$ of a solution containing 6-OHDA $(3 \mu \mathrm{g} / \mu \mathrm{l}$; RBI, USA), $0.9 \%$ saline, and $0.2 \%$ ascorbic acid was injected over a 6 -min period with a Ranzel delivery pump. The cannula was left in place for $1 \mathrm{~min}$ after the injections, and then slowly withdrawn. The same protocol was used for sham-operated rats, except that only the saline solution $(0.9 \% \mathrm{NaCl}$ plus $0.2 \%$ ascorbic acid) was injected (Espejo, 1997; Espejo and Miñano, 2001). Immediately after surgery, the rats were given an intramuscular injection of the antibiotic ceftriazone $(10 \mathrm{mg} / 0.2 \mathrm{ml})$.

Regarding experiment 2, rats were lesioned in both the mPFC and nucleus accumbens. Following a similar meth- 
odology to that explained above, the blunted cannula was also lowered to the nucleus accumbens after prefrontocortical lesion, at the following coordinates: $\mathrm{AP}= \pm 1.5 \mathrm{~mm}$, $\mathrm{L}=+1.5, \mathrm{~V}=-7$ (Paxinos and Watson, 1997). At each site, $1 \mu \mathrm{l}$ of a 6 -OHDA solution $(5 \mu \mathrm{g} / \mu \mathrm{l})$ was injected. The same protocol was used for sham-operated rats, except that only the saline solution was injected in both the mPFC and nucleus accumbens. Intra-accumbal toxin injection affected the shell and core territories of the nucleus accumbens, since a volume of $0.5 \mu \mathrm{l}$ is known to spread about a distance of $1 \mathrm{~mm}^{3}$ (Routtenberg, 1972).

\section{Behavioral Testing}

Two weeks after surgery, contextual fear conditioning and social memory tests were carried out. Regarding fear conditioning, the conditional chamber was made of transparent Plexiglas on every side, and a 36-bar insulated shock grid floor. The chamber was ever surrounded by the same spatial cues or colored objects and a background white noise $(75 \mathrm{~dB})$ was maintained throughout the experiments (conditioned stimuli, CS). The floor was removable, and after each experimental subject, it was cleaned up with alcohol. The grid floor was connected to a Shocker to deliver electric shocks. Rats were placed in the conditioning chamber for $3 \mathrm{~min}$ before the onset of the US (three electric shocks, $1 \mathrm{~mA}$ for $4 \mathrm{~s}, 10 \mathrm{~s}$ apart, offset-to-onset). This test is very appropriate for the study of memory because robust learning can be triggered with a single 3 min trial. After the last CS/US pairing, the rat was left in the conditioning chamber for another $30 \mathrm{~s}$ and the duration of freezing was quantified. A complete immobility response in an alerting posture (with respiratory movements only) was considered as freezing. Later, lesioned and sham rats were repeatedly tested at $30 \mathrm{~min}, 3 \mathrm{~h}, 1,3$, and 5 days after the conditioning test (F0.5h, F3h, F1d, F3d, F5d tests), to give an index of long-term extinction of freezing, whose absolute duration was quantified during $5 \mathrm{~min}$ in the chamber where the rats were shocked. Moreover, freezing duration was also quantified in smaller time bins (every $60 \mathrm{~s}$ ) in order to give a within-session extinction index as well as to calculate the percentage of recovery of freezing between sessions, index of extinction retention (Quirk et al, 2000). Thus, withinsession extinction was calculated as the difference of percent freezing duration between the last and the first minute of each test. Spontaneous recovery was calculated as the difference between percent freezing duration between the first minute of a test session and the last minute of the previous session. For experiment 1, separate groups of sham and lesioned rats (referred to as 'nonshocked' groups) were tested following the same protocol except for the fact that the electric shock was not delivered, in order to detect if changes in freezing in lesioned rats were because of a specific response to the aversive stimulus or a nonspecific increase relative to sham rats.

Regarding the social interaction tests, experiments were carried out in the rats' cages. Juvenile male Wistar rats (3week old, $40-50 \mathrm{~g}$ ) were isolated in individual cages $30 \mathrm{~min}$ before the experiments. An unfamiliar juvenile conspecific was introduced in the adult's cage for a first exploration period (P1, $5 \mathrm{~min})$, after which the juvenile was returned to the isolation box. The delay period was followed by a second 5-min exploration at either 30 or $120 \mathrm{~min}$ (independent groups) with the same juvenile (P30, P120 sham and lesioned groups, respectively, $n=7-9$ each). During the test, the durations of 'social interaction', 'nonsocial exploration', and 'resting' were measured. 'Social interaction' was quantified as the sum duration of sniffing, grooming, or pawing elicited by the adult rat toward the juvenile. The category 'nonsocial exploration' comprised 'walking and sniffing around the cage', 'self-grooming', and 'rearing' (upright posture). 'Resting' was defined as the rat quietly lying on the floor. During this test, a very sensitive shortterm memory test, adults recognize the juvenile at a $30-\mathrm{min}$ delay after each presentation, but after a 2-h delay the adults have forgotten it (Letty et al, 1997). Finally, in experiment 1 , separate groups of sham and lesioned rats (referred to as 'unfamiliar juvenile' groups) were tested following the same protocol, except for the fact that the juvenile was ever unfamiliar.

The rats' behavior during tests was recorded on videotape under white illumination. The tapes were later visualized and the behavior analyzed by keyboard entry to a computer programmed to perform statistical and ethological analyses. Videotapes were scored 'blind' by a highly trained observer (intrarater reliability $\geqslant 0.9$ ). All behavioral tests were carried out during the last phase of the light period (18:00-20:00 h).

\section{Postmortem Neurochemical Analysis}

Three weeks after the lesions, the rats were killed by decapitation, the brains quickly removed and placed on ice, and bilateral prefrontal cortex and nucleus accumbens immediately dissected, weighed and frozen at $-80^{\circ} \mathrm{C}$. Later, tissue samples were homogenized in $0.5 \mathrm{ml}$ of an ice-cold solution containing $0.4 \mathrm{M} \mathrm{HClO}_{4}, 0.5 \mathrm{M}$ sodium acetate, and $0.5 \mathrm{M}$ acetic acid, and centrifuged at $27000 \mathrm{rpm}$ for $60 \mathrm{~min}$ at $4{ }^{\circ} \mathrm{C}$. The supernatants were decanted and filtered through a $0.45 \mu \mathrm{m}$ filter (Sartorius), and frozen at $-80^{\circ} \mathrm{C}$ until highperformance liquid chromatography (HPLC) assay. The electrochemical performance was based essentially on the method described by Saito et al (1992). Aliquots $(10 \mu \mathrm{l})$ of each sample were injected directly into the HPLC system (System Gold, Beckman), consisting of a solvent delivery pump with a pulse-dampener, an automatic sample injector (Carnegie Medicine), and an analytical C18 reverse-phase column (Ultrasphere $3 \mu \mathrm{m}$ particle size, $75 \mathrm{~mm} \times 4.6 \mathrm{~mm}$ ID, Beckman). The ESA model 5100 A Coulochem electrochemical detection system consisted of a model 5021 conditioning cell (detector setting $+400 \mathrm{mV}$ ) followed in sequence by a model 5011 dual-electrode analytical cell (cell $1,+100 \mathrm{mV}$; cell $2,-260 \mathrm{mV}$ ). The output signal from the final electrode was amplified by a 5100 A controller and relayed to an integrator (Model 106, Beckman). The mobile phase for the separation of catecholamines and their metabolites was a mixture of $0.075 \mathrm{M} \mathrm{Na}_{2} \mathrm{HPO}_{4}, 1.2 \mathrm{mM}$ sodium heptanosulfonate, $0.097 \mathrm{mM}$ EDTA, and $8 \%$ metha$\mathrm{nol}(\mathrm{v} / \mathrm{v})$ adjusted to $\mathrm{pH}$ 3.6. The buffer solution was filtered through a $0.45 \mu \mathrm{m}$ membrane filter and degassed. The flow rate was set to $1.7 \mathrm{ml} / \mathrm{min}$ and pressure was around $2000 \mathrm{psi}$. The mobile phase was recycled for 2 weeks of continuous use before being replaced with fresh solution. The entire chromatographic system was run at ambient temperature. 
Peaks of biogenic amines and metabolites were identified by comparing the retention time of each peak in the sample solution with that in the standard solution. Dihydroxybenzylamine (DHBA) was used as internal standard for extraction variability. The program System Gold 2.01 (Beckman) was used to calculate monoamines levels in each sample. The contents of dopamine, 3,4-dihydroxyphenylacetic acid (DOPAC), homovanillic acid (HVA), NA, and serotonin (5-HT) were quantified in the MPFC, and those of dopamine and DOPAC in the nucleus accumbens. Dopamine turnover was estimated by the $\mathrm{DOPAC} / \mathrm{DA}$ ratio (calculated as pmoles).

\section{Statistics}

Neurochemical results were analyzed by using the Student's $t$-test for comparison of two independent groups. Regarding fear conditioning data, two-way ANOVA for repeated measures was used prior to post hoc Newman-Keuls test (fear conditioning, group as between variable, time after test as within variable). Sphericity of repeated measures was assessed before ANOVA treatment by using the Mauchly's $\mathrm{W}$ test for sphericity, in order to reveal that sphericity was not violated. For social interaction, two-way ANOVA was used prior to post hoc Newman-Keuls test (group and time as between variables). Since size populations were small, the data were logarithmically transformed $(\log [x])$ before ANOVA treatment if variance was found not to be homogeneous as assessed by the F test.

\section{RESULTS}

\section{Experiment 1}

Neurochemical data. Regarding dopamine levels, 6-OHDA lesion induced a profound reduction of $\mathrm{mPFC}$ DA content $(-76.8 \%, p<0.01)$. The dopamine metabolite HVA was also significantly reduced (Table 1 ), but not DOPAC, which was affected to a much lesser extent. The ratio DOPAC/DA, indicative of dopamine turnover, was found to be significantly enhanced in lesioned rats $(+260.7 \%, p<0.01)$. Prefrontocortical 6-OHDA lesion did not alter mPFC NA levels, but 5-HT levels were significantly reduced after prefrontocortical 6-OHDA lesion $(-21.7 \%, p<0.05)$.

As shown in Table 2, prefrontocortical dopamine loss was followed by a significant increase in accumbal DA $(+152.3 \% v s$ controls; $p<0.01)$ and, to a lesser extent, in its metabolite DOPAC $(+32.5 \%$ vs controls; $p<0.05)$. The DOPAC/DA ratio was found to be 0.21 in sham rats, being reduced to 0.11 in lesioned rats, indicating a diminished DA 'turnover'.

\section{Behavioral results}

Contextual fear conditioning: During the conditioning test, percent time spent on freezing was similar in sham and lesioned groups (sham, $56.4 \pm 5.1 \%$; lesioned, $60.8 \pm 5.6 \%$ ), as shown in Figure 1. A significant interaction effect for freezing duration was revealed by two-way ANOVA (df $5,60 ; \mathrm{F}=3.1, p<0.05)$. No significant differences were found with respect to percent time spent on freezing (sham, $43 \pm 4 \%$; lesioned, $38.1 \pm 3.2 \%$ ), $30 \mathrm{~min}$ after the conditioning test $(\mathrm{F} 0.5 \mathrm{~h})$ revealing that short-term fear conditioning developed in both groups. Post hoc significant differences were found in F3h, F1d, and F3d tests, because lesioned rats spent more time than sham rats displaying the freezing response at $3 \mathrm{~h}(\mathrm{~F} 3 \mathrm{~h}$ sham $=28.2 \pm 3.1 \%$; $\mathrm{F} 3 \mathrm{~h}$ lesioned $=44.2 \pm 5 \%, p<0.01), 1$ day after conditioning $($ F1d sham $=25.5 \pm 3 \%$; F1d lesioned $=40.8 \pm 4 \%, p<0.01)$ and 3 days after conditioning (F3d sham $=21.4 \pm 3 \%$; F3d lesioned $=29.8 \pm 3 \%, p<0.05$ ). Freezing was significantly reduced in sham rats during F3h, F1d, and F3d tests $v s$ the corresponding F0.5h test $(p<0.01)$. Five days after conditioning no differences were observed between both groups (F5d sham, $14.7 \pm 1 \%$; F5d lesioned, $17.7 \pm 2 \%$ ), freezing being significantly diminished in both groups $v s$ the corresponding F0.5h test $(p<0.01)$, indicating extinction of contextual fear in both groups. Regarding within-session

Table I Monoamine Levels (pg/mg Wet Weight of Tissue) in the mPFC in Sham and 6-OHDA-Lesioned Rats

\begin{tabular}{lllllll}
\hline Group & DA & DOPAC & HVA & NA & 5-HT & DOPAC/DA (pmol) \\
\hline Sham & $128.5 \pm 33.1$ & $32.4 \pm 12.1$ & $211.2 \pm 24.5$ & $184.8 \pm 35.5$ & $505.5 \pm 78.2$ & $0.28 \pm 0.1$ \\
6-OHDA & $29.8 \pm 12.2 * * * 26.8 \pm 10.2$ & $166.8 \pm 28.5 *$ & $199.3 \pm 42.2$ & $395.7 \pm 40.1 *$ & $1.01 \pm 0.2 * *$ \\
\hline
\end{tabular}

The data represent that of pooled shocked and nonshocked rats. Mean \pm SEM, $* p<0.05$, *** $<0.01$ vs sham (Student's t-test). Abbreviations: DA, dopamine; DOPAC, 3,4-dihydroxyphenylacetic acid; HVA, homovanillic acid; NA, noradrenaline; $5-\mathrm{HT}$, serotonin.

Table 2 Concentrations of Dopamine and DOPAC within the Nucleus Accumbens after Sham or 6-OHDA-Induced Lesion of the mPFC (pg/mg Wet Weight of Tissue)

\begin{tabular}{lllll}
\hline Structure & Group & DA & DOPAC & DOPAC/DA (pmol) \\
\hline Nucleus & Sham & $5779.8 \pm 445.5$ & $1090.8 \pm 110.3$ & $0.21 \pm 0.1$ \\
Accumbens & $6-O H D A$ & $14622.8 \pm 1455.9 * *$ & $1463.3 \pm 150.6 *$ & $0.11 \pm 0.1 * *$ \\
\hline
\end{tabular}

Values are mean \pm SEM. ${ }^{*} p<0.05,{ }^{*} * 0<0.0$ I vs sham group (Student's $t$-test). Abbreviations: DA, dopamine; DOPAC, 3,4-dihydroxyphenylacetic acid. 
extinction, a similar percentage of within-session extinction of freezing was observed in 'shocked' sham and lesioned rats. However, extinction retention was impaired since

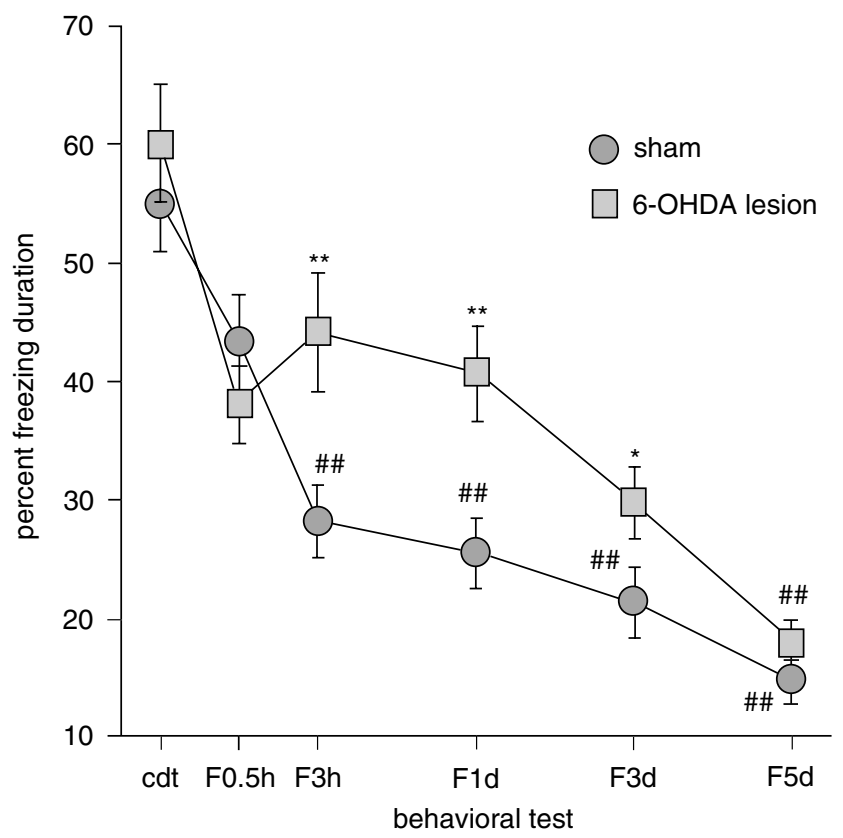

Figure I Percent time displaying freezing during fear conditioning tests in rats with dopamine loss in the mPFC. Mean \pm SEM. $* * * 0<0$. 01 vs sham group; $p<0.0$ I vs the same group during the F0.5h test. Abbreviations: $\mathrm{Cdt}$, first or conditioning test; F0.5h, F3h, FId, F3d, and F5d, 30 min, $3 \mathrm{~h}, \mathrm{I}, 3$, and 5 days after the conditioning test, respectively. All tests lasted 300 s except the conditioning one (232s). spontaneous recovery of percent freezing duration was significantly enhanced in lesioned rats during F3h and F1d tests $(p<0.01)$, as shown in Table 3 .

No differences in freezing responses were observed in the 'nonshocked' groups of sham and lesioned rats, and a significant habituation of freezing was observed in both groups during the last two tests (Table 4). All these findings indicate that there was a significant delay of extinction of contextual fear conditioning in 'shocked' rats after prefrontocortical dopamine loss.

Social interaction: As shown in Figure 2, lesioned rats spent significantly less time exploring the juvenile than sham rats during the first exposition (P1 sham $=89.8 \pm 8.7 \mathrm{~s} ; \quad \mathrm{P} 1$ lesioned $=45.8 \pm 5.4 \mathrm{~s} ; \quad p<0.01$ ), indicating that social interaction was reduced by the 6-OHDA-induced lesion. This significant difference was maintained at $30 \mathrm{~min}(\mathrm{P} 30$ sham $=57 \pm 4.3 \mathrm{~s}$; P30 lesioned $=26.8 \pm 3.2 \mathrm{~s} ; p<0.01)$. The difference between the first and second test was significant for both groups $(p<0.01)$, and the percent reduction in time spent exploring the familiar juvenile was similar (sham, $-36.5 \pm 3.1 \%$; lesioned, $-41.5 \pm 3.9 \%)$. These data indicate that shortterm social interaction memory developed in sham and lesioned rats. The time spent on social interaction was returned to basal levels at $120 \mathrm{~min}$ after the test (P120 sham $=81 \pm 6.7 \mathrm{~s} ; \mathrm{P} 120$ lesioned $=61.8 \pm 7.7 \mathrm{~s})$, and the duration of social interaction was found to be significantly different between both groups $(p<0.05)$, as well as with respect to the $30 \mathrm{~min}$ test in both groups $(p<0.01)$, indicating that short-term memory had worn off in every rat but duration of social interaction remained diminished in lesioned rats. Two-way ANOVA did not show any

Table 3 Percentage of Within-Session Extinction of Freezing as Well as That Spontaneous Recovery of Freezing between Tests (Extinction Retention) in Shocked Sham Rats and Animals with Dopamine Loss Within the mPFC

\begin{tabular}{lcclll}
\hline Group & Fo.5h & F3h & FId & F3d & F5d \\
\hline Within-session extinction & & & & \\
Sham & $10.7 \pm 1.1$ & $8.5 \pm 2$ & $8.8 \pm 0.8$ & $9.4 \pm 1.8$ & $8.9 \pm 1$ \\
6-OHDA & $9.9 \pm 1.2$ & $8.6 \pm 1.5$ & $8.6 \pm 0.9$ & $8.8 \pm 1.6$ & $9.2 \pm 1$ \\
\multicolumn{2}{l}{ Spontaneous recovery } & & & & \\
Sham & - & $-11 \pm 1.1$ & $3.2 \pm 1$ & $3.3 \pm 0.9$ & $0.2 \pm 0.1$ \\
6-OHDA & - & $10 \pm 1.1 * * * 1$ & $8.1 \pm 1 * *$ & $2.9 \pm 0.9$ & $0.5 \pm 0.1$ \\
\hline
\end{tabular}

Mean \pm SEM. *** $p<0.0$ I vs the sham group during the same test (Student's $t$-test). Within-session extinction was calculated as the difference of percent freezing duration between the fifth and the first minute of each test. Spontaneous recovery (index of extinction retention) was calculated as the difference between percent freezing duration between the first minute of the session and the last minute of the previous session. Abbreviations: F0.5h, F3h, FId, F3d, F5d, 30 min, $3 \mathrm{~h}, \mathrm{I}, 3$, and 5 days after the conditioning test, respectively.

Table 4 Percent Time Spent in Freezing in NonShocked Sham Rats and Animals with Dopamine Loss within the mPFC

\begin{tabular}{lllllll}
\hline Group & $\begin{array}{l}\text { Conditioning } \\
\text { (without shock) }\end{array}$ & F0.5h & F3h & Fld & F3d & F5d \\
\hline Sham & $15.8 \pm 2.6$ & $7.7 \pm 1.1$ & $6.5 \pm 1$ & $5.5 \pm 0.8$ & $4.3 \pm 0.8 *$ & $4.4 \pm 1^{*}$ \\
6-OHDA & $12.6 \pm 2.1$ & $8.3 \pm 1.2$ & $5.6 \pm 1.1$ & $5.6 \pm 0.9$ & $4.5 \pm 1 *$ & $4.2 \pm 0.9 *$ \\
\hline
\end{tabular}

Mean \pm SEM. * $p<0.05$ vs the same group during the F0.5 test. Abbreviations: conditioning, first or conditioning test; F0.5h, F3h, FId, F3d, F5d, 30 min, 3 h, I, 3, and 5 days after the conditioning test, respectively. 


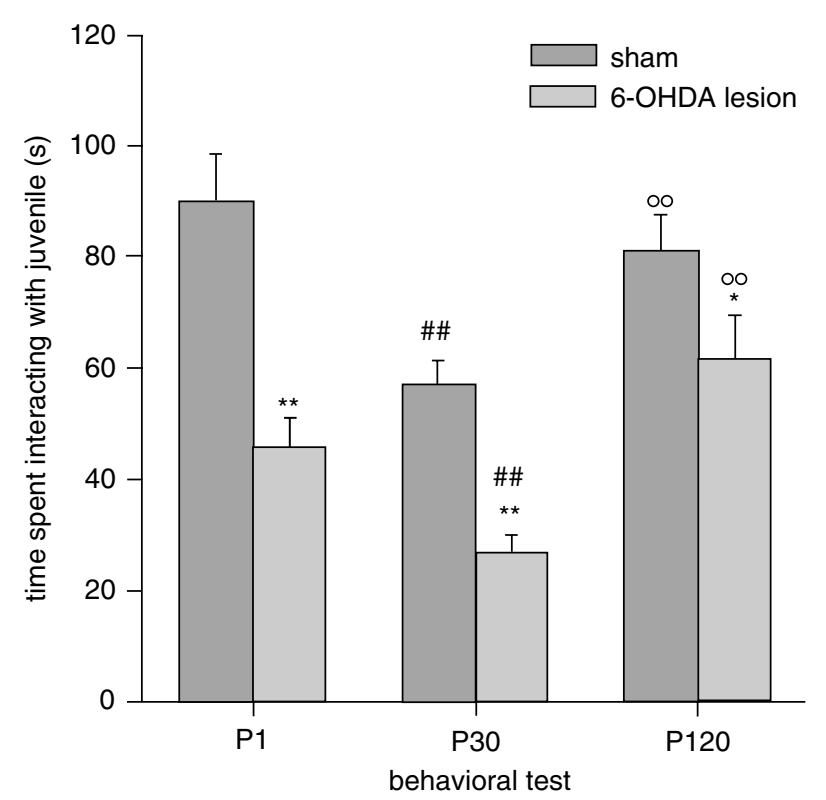

Figure 2 Time spent interacting with the juvenile during the social interaction memory tests, in rats with dopamine loss in the MPFC (familiar juvenile). Mean \pm SEM. $* p<0.05, * * * 0.01$ vs sham group, $\#$ \# $p<0.01$ vs the same group at the PI test; ${ }^{\circ} \mathrm{p}<0.0 \mathrm{I}$ vs the same group at the P30 test. Abbreviations: PI, first social interaction test; P30, PI20, at 30 and 120 min after the first social interaction test, respectively. Both groups followed a similar time-course of social interaction memory, even though time the spent on interaction was significantly diminished in the lesioned group at every time point. interaction effect, further indicating that the time course of social interaction memory was similar in both groups, even though duration of social interaction was reduced after 6OHDA lesion at every time point. Regarding 'nonsocial exploration' and 'resting', no differences between 'nonsocial exploration' were found for both groups of rats, but 'resting' was significantly enhanced in lesioned animals at every time point $(p<0.05)$, as shown in Table 5. Regarding separate sham and lesioned 'unfamiliar juvenile' groups (tested against an unfamiliar juvenile following the same protocol), the time spent by lesioned rats interacting with the juvenile was reduced at every time point ( $p<0.01 v s$ sham rats). 'Resting' was also enhanced at every time point in lesioned rats, and 'nonsocial exploration' was not significantly enhanced, confirming that social interaction was specifically reduced by the 6-OHDA-induced $\mathrm{mPFC}$ lesion leading to enhanced 'resting' behavior (Table 6).

\section{Experiment 2}

Neurochemical data. In the mPFC, 6-OHDA lesion induced a profound reduction of mPFC DA content $(-79.8 \%$, $p<0.01)$. A similar change was observed for HVA. The ratio DOPAC/DA was found to be significantly enhanced in lesioned rats $(+245.5 \%, p<0.01)$. Regarding neurochemical changes in the nucleus accumbens, 6-OHDA lesion induced a significant reduction of accumbal DA content (sham, $6125 \pm 832.2 \mathrm{pg} / \mathrm{mg} ;$ lesioned, $1470 \pm 187 \mathrm{pg} / \mathrm{mg} ;-76 \%$ reduction, $p<0.01$ ), and the dopamine 'turnover' was significantly enhanced (sham rats, ratio $=0.19$; lesioned

Table 5 Time Spent in 'NonSocial Exploration' and 'Resting' in Sham and 6-OHDA-Lesioned Rats in the mPFC during the Social Interaction Memory Test (Familiar Juvenile)

\begin{tabular}{llllc}
\hline Behavior & Group & PI & P30 & P I20 \\
\hline Nonsocial exploration & Sham & $188 \pm 15.5$ & $218 \pm 20.1 \#$ & $195 \pm 17.7$ \\
& 6-OHDA & $199 \pm 13.3$ & $221 \pm 18.8 \#$ & $203 \pm 19.4$ \\
Resting & Sham & $22.2 \pm 3.2$ & $24.6 \pm 4.5$ & $24 \pm 3.6$ \\
& 6-OHDA & $55.2 \pm 9.1 * *$ & $50.4 \pm 8.7 * *$ & $35.2 \pm 5.2 * \#$ \\
\hline
\end{tabular}

Mean \pm SEM. ${ }^{*} p<0.05,{ }^{*} * p<0.0$ I vs sham group at the same test; ${ }^{*} p<0.05$ vs the same group at the PI test. Abbreviations: PI, first interaction test; P30, PI20 at 30 and 120 min after the first interaction test, respectively. Both groups followed a similar time-course of 'nonsocial exploration', but 'resting' was enhanced at every time point in 6-OHDA-lesioned rats.

Table 6 Time Spent in 'Social Interaction', 'NonSocial Exploration', and 'Resting' in Sham and 6-OHDA-Lesioned Rats in the mPFC during the Social Interaction Memory Test (Unfamiliar Juvenile)

\begin{tabular}{lllll}
\hline Behavior & Group & PI & P30 & PI20 \\
\hline Social interaction & Sham & $101 \pm 9.8$ & $98.8 \pm 8.8$ & $82.5 \pm 9.9$ \\
& 6-OHDA & $67.8 \pm 7.8 * *$ & $59.9 \pm 5.7 * *$ & $64.7 \pm 7.6 *$ \\
Nonsocial exploration & Sham & $175 \pm 12.5$ & $178 \pm 18.1$ & $193 \pm 15.7$ \\
& 6-OHDA & $187 \pm 11.3$ & $198 \pm 19.8$ & $198 \pm 18.2$ \\
Resting & Sham & $24 \pm 3.2$ & $23.2 \pm 4.8$ & $24.5 \pm 3.3$ \\
& 6-OHDA & $45.2 \pm 8.1 * *$ & $42.1 \pm 6.7 * *$ & $37.3 \pm 5.3 *$ \\
\hline
\end{tabular}

Mean \pm SEM. $* p<0.05, * * p<0.0$ I vs sham group at the same test. Abbreviations: PI, first interaction test; P30, $\mathrm{PI20}$, at 30 and $120 \mathrm{~min}$ after the first interaction test, respectively. Both groups followed a similar time-course of 'nonsocial exploration', but 'social interaction' was reduced and 'resting' was enhanced at every time point in 6-OHDA-lesioned rats. 
Table 7 Percent Time Spent in Freezing in Sham Rats and Animals with Dopamine Loss within both the mPFC and Nucleus Accumbens

\begin{tabular}{llllcll}
\hline Group & Conditioning & F0.5h & F3h & FId & F3d & F5d \\
\hline Sham & $58.8 \pm 5.6$ & $42.6 \pm 4$ & $27.1 \pm 3 \# \#$ & $25 \pm 3.1 \# \#$ & $20.6 \pm 3 \# \#$ & $15.2 \pm 1 \# \#$ \\
6-OHDA & $60.6 \pm 6.1$ & $39.3 \pm 3.5$ & $38.1 \pm 4.1 * *$ & $32.6 \pm 3 *$ & $28.3 \pm 2 * \# \#$ & $17.1 \pm 2 \# \#$ \\
\hline
\end{tabular}

Mean \pm SEM. $* p<0.05$, *** $p<0.0$ I vs sham group at the same test; ${ }^{\# \#} p<0.0$ I vs the same group during the F0.5 test. Abbreviations: conditioning, first or conditioning test; F0.5h, F3h, FId, F3d, F5d, $30 \mathrm{~min}, 3 \mathrm{~h}, \mathrm{I}, \mathrm{3}$, and 5 days after the conditioning test, respectively.

Table 8 Time (s) Spent Interacting with the Juvenile during the Social Interaction Memory Tests in Sham Rats and Animals with Dopamine Loss within both the mPFC and Nucleus Accumbens (Familiar Juvenile)

\begin{tabular}{|c|c|c|c|}
\hline Group & $\mathbf{P I}$ & P30 & PI20 \\
\hline Sham & $91 \pm 9.1$ & $61 \pm 6.8 \# \#$ & $85 \pm 7.5^{\circ \circ}$ \\
\hline 6-OHDA & $48 \pm 5.2 * *$ & $21 \pm 2.1$ ***\#\# & $49 \pm 5.7^{* * * 0}$ \\
\hline
\end{tabular}

rats, ratio $=0.27 ; p<0.05)$. In this context, it is supposed that the increase in DA 'turnover' reflects enhanced dopamine release and metabolism in the surviving DA neurons (Espejo and Miñano, 2001).

Behavioral results. Similar results to those obtained for rats only lesioned in the mPFC were obtained, as illustrated in Tables 7 and 8. For contextual fear conditioning, the delayed extinction of contextual fear after prefrontocortical dopamine loss was not altered by added lesion of the mesoaccumbal dopaminergic system. Regarding social interaction, duration of social interaction remained diminished in lesioned rats, and this effect was not affected by added dopamine loss in the nucleus accumbens. Short-term social memory was also preserved.

\section{DISCUSSION}

The neurochemical data indicated that prefrontocortical dopamine levels fell to $23.2 \%(-76.8 \%$ reduction) after 6OHDA lesion, and dopamine turnover was enhanced threefold, likely as a reactive response because of dopamine lesion in accordance with several authors (Bubser and Schmidt, 1990; Hemby et al, 1992; Kurachi et al, 1995). Prefrontocortical serotonin levels were reduced to a much lesser extent, and noradrenergic content was not modified, an important fact because reduced noradrenergic activity after prefrontocortical dopamine loss can lead to paradoxical recovery of extracellular dopaminergic levels (Venator et al, 1999). Interestingly, dopamine and metabolite contents were enhanced in the nucleus accumbens after 6OHDA lesion, suggesting that there is an inverse relation between mesocortical and mesolimbic dopaminergic systems, as proposed by others (Carter and Pycock, 1978, 1980; Pycock et al, 1980; Glowinski et al, 1984; Grace, 1991; Roberts et al, 1994; Kolachana et al, 1995; Kurachi et al, 1995). Accumbal DA turnover was reduced after mPFC lesion, a fact that could be interpreted as reflecting a decrease in firing of DA neurons projecting to nucleus accumbens after DA lesion of the MPFC, in accordance with electrophysiological studies (Harden et al, 1998). The findings support the hypothesis that a hyperdopaminergic tone emerges in the nucleus accumbens after prefrontocortical dopamine loss. It is possible that reactive neurochemical changes after prefrontocortical dopamine loss could also be detected in other sites connected with the mPFC such as dorsal striatum or midbrain areas, a topic that requires further research. Furthermore, an interaction between the experience of shocking and prefrontal lesion upon DA concentrations in shocked rats cannot be ruled out, a fact that also needs to be investigated further.

Regarding fear conditioned responses, the data indicated that acquisition and short-term memory of contextual fear conditioning was not impaired by 6-OHDA lesion within the mPFC. This finding is similar to that found for cued fear conditioning acquisition after prefrontal dopamine loss (Morrow et al, 1999) or mPFC lesion (Gewirtz et al, 1997; Quirk et al, 2000), and strongly indicates that acquisition and short-term fear conditioning memory (cued or contextual) is not dependent on the dopaminergic activity of the mPFC. However, long-term extinction of contextual fear conditioning was reliably delayed in 'shocked' lesioned rats. This effect was not because of a performance deficit because: (i) freezing was not enhanced in 'nonshocked' lesioned rats, and (ii) although lesioned rats displayed more 'resting' behavior in the social interaction tests, this immobility response is quite different in nature to 'freezing' that represents an alerting response rather than a quietly resting behavior. Prefrontocortical lesions were also associated with a normal within-session extinction, but spontaneous recovery between tests was significantly enhanced in lesioned rats, pointing to impaired extinction retention after dopamine depletion of the mPFC. In this context, the extinction of cued fear conditioning is also delayed after dopamine depletion of the mPFC (Morrow et al, 1999), and no changes in within-session extinction along with impaired extinction retention have been reported after mPFC lesions 
(Quirk et al, 2000), revealing that similar changes on extinction are found in both types of conditioned fear after prefrontal dopamine loss, even though both types of fear conditioning rely on different neural systems (Phillips and Le Doux, 1992; Kim et al, 1993). The data indicate that a normal dopamine activity within the prefrontal cortex is critical in coordinating the normal long-term extinction of fear conditioning, but mesoprefrontal dopamine neurons are not involved in the acquisition, short-term memory or within-session extinction of fearful behaviors. Contextual fear conditioning, unlike cued conditioning, involves hippocampal function (Phillips and Le Doux, 1992; Kim et al, 1993), and the mPFC has extensive bidirectional connections with the hippocampus (Goldman-Rakic et al, 1984; von Hoesen et al, 1972, 1975), apart from inputs to the amygdala and midbrain sites that mediate fear responses (Sesack et al, 1989), indicating that dopamine activity in the mPFC could modulate the neural interaction between these areas involved in long-term memory of contextual fear conditioning. Finally, the normal acquisition and delayed long-term extinction of fear conditioning were not modified after added accumbal dopamine loss, even though it is known that dopamine metabolism is enhanced in the nucleus accumbens during conditioned fear (Morrow et al, 1996, 1999), indicating that this neurochemical change appears not to mediate contextual fear conditioning responses.

Regarding social interaction, the development of shortterm social interaction memory was not altered after prefrontocortical dopamine loss, as revealed by parallel time courses of social interaction memory for both sham and lesioned rats. It is evident that if time-course of social interaction memory was normal, there was clearly acquisition. Then, it can be concluded that both acquisition and short-term social interaction memory were not altered by prefrontal dopamine loss. However, the time spent on social interaction was reliably diminished in lesioned rats, a finding that is clearly in contrast with the fact that prefrontal transection enhances social interaction in rats (Gonzalez et al, 2000; Tucci et al, 2000), suggesting that neurotransmitter systems other than the mPFC dopaminergic one are involved in this effect. The reduction of social interaction is difficult to explain, but it could be accounted for by a low attention level or disrupted cognitive processes, which are known to emerge after prefrontal dopamine loss (Lavielle et al, 1979; Herman et al, 1982), leading to lower interest for exploring a conspecific. However, lesioned rats did not show an attentional deficit as revealed by normal (or augmented) responses in the contextual fear conditioning experiment, pointing to a cognitive deficit altering the normal performance in the social interaction task. It is known that the mPFC is critically involved in social interaction performance (Chaillan et al, 1997; Gonzalez et al, 2000; Tucci et al, 2000), and that there is a clear relation between prefrontal dopamine and 'cognitive performance' (Roberts et al, 1998); hence it can be hypothesized that an intact $\mathrm{mPFC}$ dopaminergic network is important for a normal social interaction activity. Resting behavior was increased in parallel to the reduction of social interaction in lesioned rats, a fact that could also be related to low interest in exploring a conspecific. Since serotonergic mPFC hypoactivity is known to reduce exploratory behavior as a whole (Lipska et al, 1992), a role of added serotonergic mPFC hypoactivity on the enhancement of resting in lesioned rats cannot be ruled out. Changes in social interaction were not altered after added dopamine loss within the nucleus accumbens. In this context, dopamine neurotransmission in the nucleus accumbens is known to be enhanced during social interaction in normal rats (Gonzalez et al, 2000; Tucci et al, 2000), but this neurochemical change appears not to mediate the time spent on social interaction according to the results of the present study.

In summary, the findings of the present study indicate that prefrontocortical dopamine loss leads to: (i) a delayed long-term extinction of contextual fear conditioning and impaired extinction retention without changes in acquisition and short-term contextual fear conditioning, (ii) reduction of time spent in social interaction without alteration of acquisition and short-term social interaction memory, and (iii) a reactive increase in dopaminergic activity in the nucleus accumbens without functional implication on observed changes.

\section{ACKNOWLEDGMENTS}

This study was supported by grants from Spanish DGES (PM98-015) and Plan Andaluz de Investigacion (CVI-127). The author thanks Dr J Miñano (Sevilla, Spain) for HPLC measurements, and Mr Antonio León (Sevilla, Spain) for animal care.

\section{REFERENCES}

Bubser M, Schmidt WJ (1990). 6-Hydroxydopamine lesion of the rat prefrontal cortex increases locomotor activity, impair acquisition of delayed alternation tasks, but does not affect uninterrupted task in the radial maze. Behav Brain Res 37: 157168.

Carter CJ, Pycock CJ (1978). Lesions of the frontal cortex of the rat: changes in neurotransmitter systems in sub-cortical regions. $\mathrm{Br} \mathrm{J}$ Pharmacol 64: 430P.

Carter CJ, Pycock CJ (1980). Behavioral and biochemical effects of dopamine and noradrenaline depletion within the medial prefrontal cortex of the rat. Brain Res 192: 163-176.

Chaillan F, Marchetti E, Roman F, Delfosse F, Soumireu-Mourat B (1997). Opposite effects depending on learning and memory demands in dorso-medial prefrontal cortex lesioned rats performing an olfactory task. Behav Brain Res 82: 203-212.

Dantzer R, Bluthé RM, Koob GF, Le Moal M (1987). Modulation of social memory in male rats by neurohypophyseal peptides. Psychopharmacology 91: 363-368.

Espejo EF (1997). Selective dopamine depletion within the medial prefrontal cortex induces anxiogenic-like effects in rats placed on the elevated plus-maze. Brain Res 762: 281-284.

Espejo EF, Miñano J (2001). Adrenergic hyperactivity and metanephrine excess in the nucleus accumbens after prefrontocortical dopamine depletion. J Neurophysiol 85: 1270-1275.

Gewirtz JC, Falls WA, Davis MD (1997). Normal conditioned inhibition and extinction of freezing and fear potentiated startle following electrolytic lesions of medial prefrontal cortex. Behav Neurosci 111: 712-726.

Glowinski J, Tassin JP, Thierry AM (1984). The mesocortico-prefrontal dopaminergic neurons. Trends Neurosci 7: $415-418$. 
Goldman-Rakic PS, Selemon LD, Schwartz ML (1984). Dual pathways connecting the dorsolateral prefrontal cortex with the hippocampal formation and parahippocampal cortex in rhesus monkey. Neuroscience 12: 719-743.

Gonzalez LE, Rujano M, Tucci S, Paredes D, Silva E, Alba G et al (2000). Medial prefrontal transection enhances social interaction. I: behavioral studies. Brain Res 887: 7-15.

Grace AA (1991). Phasic versus tonic dopamine release and the modulation of dopamine system responsivity: a hypothesis for the ethiology of schizophrenia. Neuroscience 41: 1-24.

Harden DG, King D, Finlay J, Grace AA (1998). Depletion of dopamine in the prefrontal cortex decreases the basal electrophysiological activity of mesolimbic dopamine neurons. Brain Res 794: 96-102.

Hemby SE, Jones GH, Neill DB, Justice JB (1992). 6-Hydroxydopamine lesions of the medial prefrontal cortex fail to influence cocaine-induced place conditioning. Behav Brain Res 49: 225-230.

Herman JP, Guillonneau D, Dantzer R, Scatton B, SmerdjianRouquier L, Le Moal M (1982). Differential effects of inescapable footshocks and of stimuli previously paired with inescapable footshocks on dopamine turnover in cortical and limbic areas of the rat. Life Sci 30: 2207-2214.

Kim JJ, Fanselow MS (1992). Modality-specific retrograde amnesia of fear. Science 256: 675-677.

Kim JJ, Fanselow MS, DeCola JP, Fernandez JL (1993). Selective impairment of long-term but not short-term conditioned fear by the $N$-methyl-D-aspartate antagonist APV. Behav Neurosci 106: 591-596.

Kolachana BS, Saunders RC, Weinberger DR (1995). Augmentation of prefrontal cortical monoaminergic activity inhibits dopamine release in caudate nucleus: an in vivo neurochemical assessment in the rhesus monkey. Neuroscience 69: 859-868.

Kowalska DM, Bachevalier J, Mishkin M (1991). The role of the inferior prefrontal convexity in performance of delayed nonmatching-to-sample. Neuropsychologia 29: 583-600.

Kurachi M, Yasui SI, Kurachi T, Shibata R, Murata M, Hagino H et al (1995). Hypofrontality does not occur with 6-hydroxydopamine lesions of the medial prefrontal cortex in rat brain. Eur $J$ Neuropsychopharmacol 5: 63-68.

Lavielle S, Tassin JP, Thierry AM, Blanc G, Herve D, Barthelemy C et al (1979). Blockade by benzodiazepines of the selective high increase in dopamine turnover induce by stress in mesocortical dopaminergic neurons of the rat. Brain Res 168: 585-594.

Letty S, Child R, Dumuis A, Pantaloni A, Bockaert J, Rondouin G (1997). 5-HT4 receptors improve social olfactory memory in the rat. Neuropharmacology 36: 681-687.

Levy R, Goldman-Rakic PS (2000). Segregation of working memory functions within the dorsolateral prefrontal cortex. Exp Brain Res 133: 23-32.

Lipska BK, Jaskiw GE, Arya A, Weinberger DR (1992). Serotonin depletion causes long-term reduction of exploration in the rat. Pharmacol Biochem Behav 43: 1247-1252.

Morgan MA, LeDoux JE (1995). Differential contribution of dorsal and medial prefrontal cortex to acquisition and extinction of conditioned fear in rats. Behav Neurosci 109: 681-688.

Morgan MA, Romaski LM, LeDoux JE (1993). Extinction of emotional learning: contribution of medial prefrontal cortex. Neurosci Lett 163: 109-113.

Morrow BA, Elsworth JD, Rasmusson AM, Roth RH (1999). The role of mesoprefrontal dopamine neurons in the acquisition and expression of conditioned fear in the rat. Neuroscience 92: 553 564.

Morrow BA, Elsworth JD, Roth RH (1996). Tyrosine enhances behavioral and mesocortical dopamine responses to aversive conditioning. Synapse 22: 100-105.

Murphy BL, Arnsten AFT, Goldman-Rakic PS, Roth RH (1996). Increased dopamine turnover in the prefrontal cortex impairs spatial working memory in rats and monkeys: pharmacological reversal of stress induced impairment. J Neurosci 16: 7768-7775.

Paxinos G, Watson C (1997). The Rat Brain in Stereotaxic Coordinates. Academic Press: Sydney.

Pério A, Terranova JP, Worms P, Bluthé RM, Dantzer R, Bizière K (1989). Specific modulation of social memory in rats by cholinomimetic and nootropic drugs, by benzodiazepine inverse agonists, but not by psychostimulants. Psychopharmacology 97: 262-268.

Phillips RG, Le Doux JE (1992). Differential contribution of amygdala and hippocampus to cued and contextual fear conditioning. Behav Neurosci 106: 274-285.

Pycock CJ, Kerwin RW, Carter CJ (1980). Effect of lesion of cortical dopamine terminals on subcortical dopamine receptors in rats. Nature 286: 74-77.

Quirk GJ, Russo GK, Barron JL, Lebron K (2000). The role of ventromedial prefrontal cortex in the recovery of extinguished fear. J Neurosci 20: 6225-6231.

Roberts AC, De Salvia MA, Wilkinson LS, Collins P, Muir JL, Everitt BJ et al (1994). 6-Hydroxydopamine lesions of the prefrontal cortex in monkeys enhance performance on an analogue of the Wisconsin Card Sorting test: possible interactions with subcortical dopamine. J Neurosci 17: 4829-4838.

Roberts AC, Robbins TW, Weiskratz L (1998). Discussions and conclusions. In: Roberts AC, Robbins TW, Weiskrantz L (eds). The Prefrontal Cortex: Executive and Cognitive Functions. Oxford University Press: Oxford. pp 221-242.

Routtenberg A (1972). Intracranial chemical injection and behavior: a critical review. Behav Biol 7: 601-641.

Saito H, Murai S, Abe E, Masuda Y, Itoh T (1992). Rapid and simultaneous assay of monoamine neurotransmission and their metabolites in discrete brain areas of mice by HPLC with coulometric detection. Pharmacol Biochem Behav 42: 351-356.

Sesack SR, Deutch AY, Roth RH, Bunney BS (1989). Topographical organization of the efferent projections of the medial prefrontal cortex in the rat: an anterograde tract-tracing study with Phaseolus vulgaris leucoagglutinin. J Comp Neurol 290: 213-242.

Tucci S, Contreras Q, Paez X, Gonzalez L, Rada P, Hernandez L (2000). Medial prefrontal transection enhances social interaction. II: neurochemical studies. Brain Res 887: 259-265.

Venator DK, Lewis DA, Finlay JM (1999). Effects of partial dopamine loss in the medial prefrontal cortex on local stressevoked extracellular dopamine concentrations. Neuroscience 93: 497-505.

Von Hoesen GW, Pandya DN, Butters N (1972). Cortical afferents to the entorhinal cortex of rhesus monkey. Science 175: 14711473.

Von Hoesen GW, Pandya DN, Butters N (1975). Some connections of the entorhinal (area 28) and perirhinal (area 35) cortices of the rhesus monkey: II. Frontal lobe afferents. Brain Res 95: 25-38. 